Doug Geisler, Eva K. Grebel, and Dante Minniti, eds.

\title{
New Cluster Candidates in Dwarf Galaxies of the Local Group
}

\author{
Mario H. Pedreros \\ Departamento de Física, Facultad de Ciencias, Univ. de Tarapacá, \\ Casilla 7-D, Arica, Chile. e-mail: mpedrero@uta.cl \\ Carme Gallart \\ Andes Fellow. Departamento de Astronomía, Univ. de Chile, Casilla \\ 36-D, Santiago, Chile and Department of Astronomy, Yale Univ., P.O. \\ Box 208101, New Haven, CT 06520, USA, e-mail: cgallart@das.uchile.cl
}

\begin{abstract}
V and I images of the nearby dIrr galaxies Sextans A and Sextans B were searched for star clusters. The candidates were selected, from a sample of non-stellar objects, on the basis of their morphology, their integrated colors and magnitudes. One of the candidates in Sextans A shows a few resolved stars whose location in the color-magnitude diagram indicates the cluster is at least older than 1 Gyr.
\end{abstract}

\section{Introduction}

Within the Local Group (LG), only the dwarf irregulars (dIrr) NGC 6822 (Wyder, Hodge, \& Zucker 2000) and WLM (Hodge, Dolphin, \& Smith 1999) are known to contain globular clusters older than 1 Gyr. Several LG dIrr, however, have not yet been explored for star clusters. Using new CCD image material, we have started a search program for star clusters in dwarf galaxies of the LG. The first targets were the nearby dIrr Sextans A and B. For them, our preliminary results indicate that there are several objects that appear as globular cluster candidates. If some of these objects turned out to be real globular clusters, we would be able to compare their ages to the old halo globulars in our Galaxy and the LMC, to investigate whether or not Sextans A and B share similar formation histories with the most luminous members of the LG, in spite of being intrinsically much fainter and isolated in the outer parts of the LG.

\section{Selection of Cluster Candidates}

We obtained images of three fields each for Sextans A and Sextans B with the du Pont 100-inch Telescope at the Las Campanas Observatory, under very good seeing conditions. Out of these, one $8.8^{\prime} \times 8.8^{\prime}$ field is centered on each galaxy, and the other two are forming an L shape such as to sample their most external parts. The observations and data reduction, as well as the search for candidate star clusters, are described in detail in Pedreros \& Gallart (2001). 
In short, we first selected a sample of non-stellar objects (those with DAOPHOT indices SHARP $\geq 0.3$ and FWHM > seeing) with compact, bright and round appearance and mostly isolated. Objects similar and close to others that looked like galaxies forming a physical group were rejected. We limited our search to objects brighter $(\mathrm{V} \leq 21.6)$ than the tip of the red-giant branch (RGB) in order to only detect relatively massive star clusters. This will allow us to obtain accurate parameters for our candidates and to expect some meaningful results in a subsequent spectroscopic follow-up.

The type of objects selected in this way are most likely rich globular clusters, background E/S0 galaxies and blue compact galaxies (BCG). We detected a relatively large number of very compact and spherically symmetric red objects. A fraction of them are redder than $(\mathrm{V}-\mathrm{I})=1.2$, which is the reddest color expected for an old, metal poor single stellar population (Salasnich et al. 2000). We are currently investigating the nature of these objects, and whether they may correspond to bona-fide stellar cluster candidates by considering: a) the maximum amount of internal interstellar reddening that can be expected in these galaxies, b) the distribution of the reddest objects in the field to determine whether an excess is found closer to the galaxies and c) the colors and magnitudes expected for background galaxies.

\section{A Globular Cluster Candidate in Sextans A}

Figure 1 shows an enlargement of the most conspicuous candidate found in this search for Sextans A. Its color and magnitude (V-I = $0.95 \mathrm{mag}$ and $\mathrm{M}_{I}=-9.19$ mag) make it photometrically similar to the brightest globular clusters found, within the same color range, in the LG galaxies NGC 6822 (HVII; Wyder et al. 2000), M 33 (r12,r13,r14,r15 and u49; Christian \& Schomer 1988), NGC 185 and NGC 205 (Kim et al. 2001). Figure 2 shows a color-magnitude diagram of Sextans A where isolated stars of the candidate shown in Figure 1 have been marked with crosses. Two Padova isochrones for $\mathrm{Z}=0.0004$ and ages 2 and 15 Gyr (Bertelli et al. 1994) are also shown in the Figure. The location of the stars in the color-magnitude diagram indicates that the cluster is at least older than 1 Gyr. This fact in itself is interesting since in a galaxy that shows an important amount of current star formation, like Sextans A, one might expect to find young massive clusters instead of a rich old cluster, as we found.

\section{References}

Bertelli, G., Bressan, A., Chiosi, C., Fagotto, F., \& Nasi, E. 1994, A\&AS, 106, 275

Christian, C.A., \& Schommer, R.A.. 1998, AJ, 95, 704

Hodge, P.W., Dolphin, A.E., \& Smith, T.R. 1999, ApJ, 521, 577

Kim, S.C. et al. 2001, in preparation

Pedreros, M.H., \& Gallart, C. 2001, in preparation

Salasnich, B., Girardi, L, Weiss, A., \& Chiosi, C. 2000, A\&A, 361, 1023

Wyder, T.K., Hodge, P.W., \& Zucker, D.B. 2000, PASP, 112, 1162 


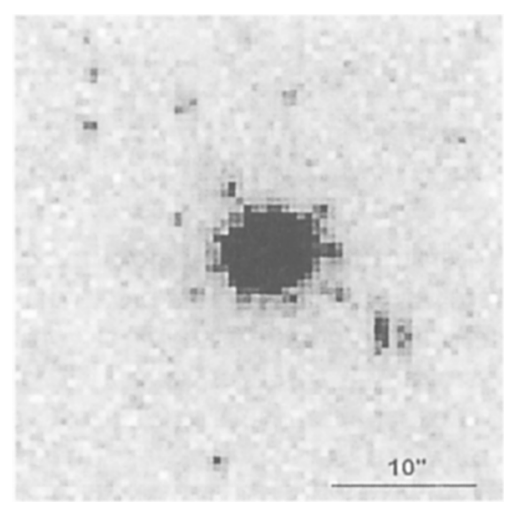

Figure 1. Enlargement of the most conspicuous cluster candidate in Sextans A.

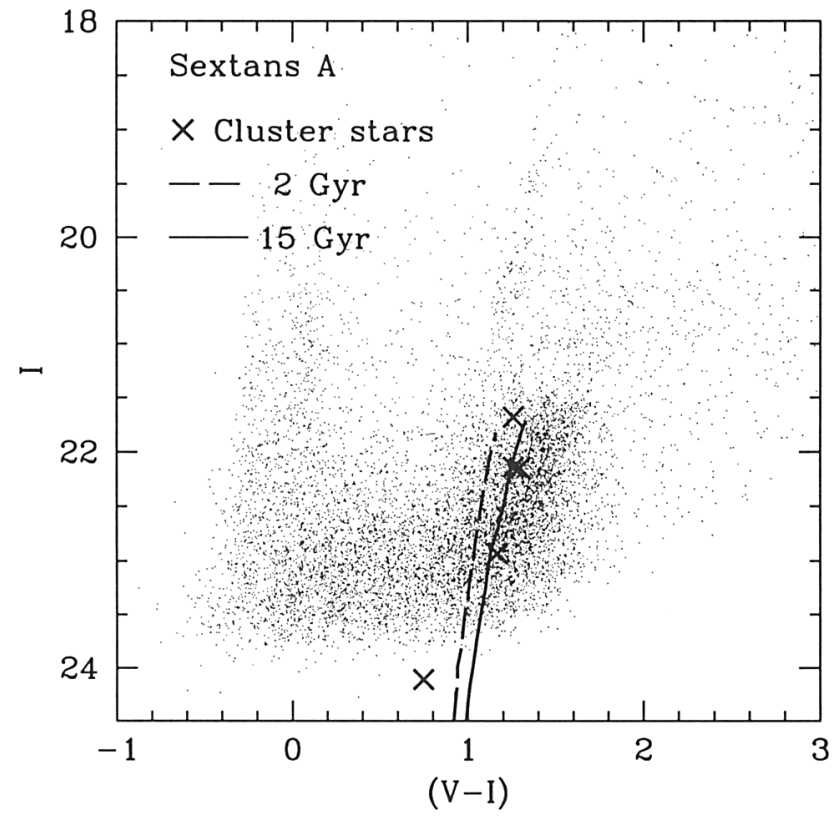

Figure 2. Color-magnitude diagram of Sextans A showing the location (crosses) of resolved stars in the cluster candidate shown in Figure 1. Two isochrones, for $\mathrm{Z}=0.0004$ and ages 2 and $15 \mathrm{Gyr}$, are also shown. 